\title{
Extensive transmission of isoniazid resistant M. tuberculosis and its association with increased multidrug-resistant TB in two rural counties of eastern China: A molecular epidemiological study
}

\author{
Yi Hu${ }^{1}$, Sven Hoffner ${ }^{2}$, Weili Jiang ${ }^{1}$, Weibing Wang ${ }^{1}$, Biao Xu ${ }^{1 *}$
}

\begin{abstract}
Background: The aim of this study was to investigate the molecular characteristics of isoniazid resistant Mycobacterium tuberculosis (MTB), as well as its contribution to the dissemination of multi-drug resistant TB (MDR-TB) in rural areas of eastern China.

Methods: A population-based epidemiological study was conducted in two rural counties of eastern China from 2004 to 2005. In total, 131 isoniazid resistant MTB isolates were molecularly characterized by DNA sequencing and genotyped by IS6110 restriction fragment length polymorphism (RFLP) and spoligotyping.

Results: The katG315Thr mutation was observed in 74 of 131 isoniazid resistant isolates and more likely to be MDR-TB (48.6\%) and have mutations in rpoB gene (47.3\%). Spoligotyping identified $80.2 \%$ of isoniazid resistant MTB isolates as belonging to the Beijing family. Cluster analysis by genotyping based on IS6110 RFLP, showed that 48.1\% isoniazid resistant isolates were grouped into 26 clusters and katG315Thr mutants had a significantly higher clustering proportion compared to those with katG wild type (73\%.vs.18\%; OR, 12.70; 95\%Cl, 6.357-14.80). Thirty-one of the 53 MDR-TB isolates were observed in 19 clusters. Of these clusters, isoniazid resistance in MDR-TB isolates was all due to the katG315Thr mutation; 18 clusters also contained mono-isoniazid resistant and other isoniazid resistant isolates.
\end{abstract}

Conclusions: These results highlighted that isoniazid resistant MTB especially with katG315Thr is likely to be clustered in a community, develop extra resistance to rifampicin and become MDR-TB in Chinese rural settings.

\section{Background}

Isoniazid (INH) is one of the most effective and specific agents for the treatment of the disease caused by $\mathrm{Myco-}$ bacterium tuberculosis (MTB). It is a cornerstone of the modern short-course chemotherapy for tuberculosis, and also widely used to treat the latent MTB infection (LTBI) to prevent the active disease and the subsequent TB transmission.

However, recent increases both in INH-resistant and multidrug-resistant (MDR) tuberculosis have been jeopardizing the efforts of global TB control through the implementation of the Directly Observed Treatment,

\footnotetext{
* Correspondence: bxu@shmu.edu.cn
'Department of Epidemiology, School of Public Health, Fudan University,

* Correspondence: bxu@shmu.edu.cn
'Department of Epidemiology, School of Public Health, Fudan University, Shanghai, China
}

(c) $2010 \mathrm{Hu}$ et al; licensee BioMed Central Ltd. This is an Open Access article distributed under the terms of the Creative Commons Attribution License (http://creativecommons.org/licenses/by/2.0), which permits unrestricted use, distribution, and reproduction in any medium, provided the original work is properly cited.

Short course (DOTS) [1,2]. In China, DOTS program has been adopted since early 1990 s, but the increased prevalence of drug resistant TB has become a significant challenge for TB control in last ten years. The prevalence of MDR-TB and INH resistant TB was estimated as $8.3 \%$ and $41.2 \%$ respectively among all cases in China, which were both high compared to the global estimates of $4.8 \%$ and $13.3 \%$ [3].

The development of INH resistance is a common first step in the evolution to MDR [4]. Thus, there has been considerable interest in both identifying the molecular basis of INH resistance and understanding the transmission pattern of the INH resistant MTB strain.

Resistance to INH is mediated by mutations in different genes in MTB, with katG [5] and inhA [6] being the 
most common. The mutation in the katG gene is a major mechanism of INH resistance in MTB [7-9]. The most common mutation is the Ser315Thr substitution in the katG gene, which is present in approximately 50$90 \%$ of all INH-resistant isolates and is associated with relatively high-level resistance to INH [9]. The mutation in $i n h A$ or its promoter region can cause $21-24 \%$ of INH resistance, with the promoter mutation (mainly in the inhA-15 position) being more common than the mutation in the structural gene [8]. A study of INH resistance in MTB has reported that a certain INH resistant strain with the katG315Thr mutation had a strong association with the development of MDR, and the successful transmission of MDR MTB as well [10].

Based on the above knowledge and findings, we hypothesized that specific INH resistant MTB strains could perpetuate the epidemic of MDR-TB in high TB burden countries like China.

Here we presented an in-depth study to determine the molecular basis of INH-resistant MTB and the transmission pattern of INH resistant MTB circulating in two rural counties of eastern China. We applied direct DNA sequencing on hotspots of katG and inhA genes/promoters as well as the IS6110 restriction fragment length polymorphism (RFLP) genotyping technique to analyze the level of clustering or recent transmission of INHresistant MTB. Combined with epidemiological evidence, the molecular information obtained from this study could give us a better understanding of the possible mechanism behind the high prevalence of MDR-TB in rural China.

\section{Methods \\ Study population}

The epidemiological methods and study population of this study were described previously [11]. It is a population-based cross-sectional study carried out in two rural counties in eastern China, i.e., Deqing County in Zhejiang Province and Guanyun County in Jiangsu Province. All TB patients notified in the County TB dispensaries within one year were enrolled in the study (Deqing, from April 1, 2004 to March 31, 2005; Guanyun, from June 1, 2004 to May 31, 2005).

\section{Drug susceptibility test}

All MTB isolates were tested for drug susceptibility using the proportion method on egg-based LJ medium [12]. The critical drug concentration were $0.2 \mu \mathrm{g} / \mathrm{ml}$ for isoniazid (INH), $40 \mu \mathrm{g} / \mathrm{ml}$ for rifampicin (RIF), $4 \mu \mathrm{g} / \mathrm{ml}$ for streptomycin (STR) and $2 \mu \mathrm{g} / \mathrm{ml}$ for ethambutol (EMB). Resistance was defined as the growth of more than $1 \%$ colonies compared to the drug free control. MDR was defined as drug resistance to at least INH and RIF. Poly drug-resistance was defined as resistance to more than one of the tested anti-TB drugs but not to both INH and RIF simultaneously.

\section{DNA sequencing}

All isolates available were investigated for the presence of the hotspot mutations related to drug resistance by direct DNA sequencing. The hotspot genes included rpoB, $k a t G$ genes and the $i n h A$ promoter region, corresponding to the drug resistance to RIF and INH respectively. The Primers pairs were CCCATGGCCGCGGCGGTCGACATT and CGCCGTCCTTGGCGGTGTATTGCC for $k a t G$ gene (GeneBank: X68081), and CCTCGCTGCCCAGAAAGGGA and ATCCCCCGGTTTCCTCCGGT for inhA promoter region (GeneBank: U41388), and GGGAGCGGATGACCACCCA and GCGGTACGGCGTTTCGATGAAC for rpoB gene (GeneBank: L27989). Mutations in these genes were determined by amplification of the corresponding hotspot DNA region by PCR followed by direct DNA sequencing with ABI 3770 DNA sequencer (Applied Biosystems, Inc., Foster City, CA, USA). The new alleles were confirmed by further PCR and re-sequencing from the original DNA. Sequencing data was independently analyzed by two biologists for quality control purposes.

\section{IS6110-RFLP genotyping}

IS6110 RFLP was done on the INH resistant isolates according to the standard protocol of van Embden et al, with data analyzed by the Gel Compar software (version 4.6, Applied Maths, Belgium) [13]. A cluster is defined as two patient MTB isolates harboring the identical IS6110 RFLP pattern. Unique strains denote unparallelness of the IS6110 RFLP profiles in the study collection. Typically, clustered strains indicate recent transmission while unique strains indicate reactive disease from a remote infection.

\section{Spoligotyping}

Spoligotyping was carried out by using the commercial kit from Isogen Bioscience BV (Maarssen, The Netherlands) [10]. The INH resistant strain clades were determined by comparison of the spoligotyping pattern with the SpolDB3 database [14] in the SpotClust program (available in http://cgi2.cs.rpi.edu/ bennek/SPOTCLUST.html) and assigned with the corresponding spoligotype international type (SIT). The Beijing family MTB was defined as the strain that hybridized only to the last nine spacer oligonucleotides (spacers 35 to 43).

\section{Statistics analysis}

SPSS software (SPSS Inc., Chicago, IL, USA) was utilized for the statistics analysis. Clinical and bacteriological characteristics were compared between katG315Thr alleles and other $\mathrm{INH}$ resistant isolates using the 
Mantel-Haenszel chi-square test. Binary logistic regression model was used for univariate and multivariate analysis to qualify and quantify the difference in clustering proportion between groups of subjects with different socio-demographic and clinical characteristics. The adjusted Odds Ratio (OR) and 95\% confidence interval (CI) were calculated by adjusting for the possible confounders (age, county and sex).

The study was approved by the Institutional Review Board of Fudan School of Public Health. Written informed consent was obtained from all the participants.

\section{Results}

In total, drug resistance profile and baseline information were successfully obtained from 399 pulmonary TB patients, 182 in Deqing and 217 in Guanyun during the study period. Of the $399 \mathrm{MTB}$ isolates from these patients, $251(62.9 \%)$ were resistant to at least one of $1^{\text {st }}$ line anti-TB drugs. Resistance to INH was the most common form of drug resistance (140/251) with a majority of cases mono-resistant to INH (55/140). Resistance to RIF was observed in 65 isolates, only 2 of which were mono-resistant. A total of 58 isolates were resistant to INH and RIF simultaneously. The details of the resistance have been reported previously [11].

DNA samples were successfully extracted from 131 of the $140 \mathrm{INH}$ resistant isolates. Of the 131 isolates, 80 were from patients who were previously diagnosed with TB and 51 were from the patients newly diagnosed. Regarding the drug resistance profile, apart from the 55 INH mono-resistant isolates, 53 were also resistant to RIF and referred to as MDR-TB; 23 were poly-resistant $(\mathrm{INH}+\mathrm{STR}$ and/or EMB) (Table 1$)$.

Table 1 Drug susceptibility profile of INH resistant MTB isolates from the study sites

\begin{tabular}{cccc}
\hline $\begin{array}{c}\text { Drug } \\
\text { resistance } \\
\text { profile }\end{array}$ & $\begin{array}{c}\text { Total } \\
\boldsymbol{n}=131\end{array}$ & \multicolumn{2}{c}{$\begin{array}{c}\text { No. of isolates } \\
\text { from patients with: }\end{array}$} \\
\cline { 3 - 4 } & & $\begin{array}{c}\text { newly } \\
\text { diagnosed TB } \\
\boldsymbol{n} \mathbf{= 8 0}\end{array}$ & $\begin{array}{c}\text { previously } \\
\text { diagnosed TB } \\
\boldsymbol{n}=\mathbf{5 1}\end{array}$ \\
\hline H & 55 & 30 & $\mathbf{2 5}$ \\
MDR-TB & 53 & 33 & 20 \\
HR & 32 & 21 & 11 \\
HRS & 12 & 6 & 6 \\
HRE & 5 & 3 & 2 \\
HRSE & 4 & 3 & 6 \\
PDR-TB & 23 & 17 & 4 \\
HS & 13 & 9 & 1 \\
HE & 2 & 1 & 1 \\
HSE & 8 & 7 &
\end{tabular}

NOTE: $\mathrm{H}$, isoniazid; $\mathrm{R}$, rifampicin; $\mathrm{S}$, streptomycin; $\mathrm{E}$, ethambutol; MDR-TB, multidrug-resistant tuberculosis; PDR-TB, polydrug-resistant tuberculosis.
DNA sequencing demonstrated that 107 of $131 \mathrm{INH}$ resistant isolates had mutations either in the katG gene or in the $i n h A$ promoter (Table 2): 61.8\% (81/131) of the isolates had a mutation in the position 315 of katG, of which $56.5 \%(74 / 131)$ INH resistant isolates contained the katG Ser315Thr nucleotide substitution. katG315Arg and katG315Asn mutations were detected in 3 and 4 isolates respectively. In addition, 28 of $131 \mathrm{INH}$ resistant isolates $(21.4 \%)$ presented the mutation in the inhA promoter region, all of which possessed the inhA$15 \mathrm{C} \rightarrow \mathrm{T}$ nucleotide substitution. Two INH resistant isolates shared the mutation in kat $G$ and inhA gene simultaneously: one with katG $315 \mathrm{Thr}$ and inhA-15T mutations and the other with katG315Arg and inhA$15 \mathrm{~T}$ mutations. No nucleotide substitutions were identified in $220 \mathrm{INH}$ sensitive isolates either at the katG315 position or the $i n h A-15$ position. The frequency of the katG315Thr mutation was significantly higher in MDRTB compared to the other drug resistant form of MTB isolates (67.9\%.vs.48.7\%; $\left.\chi^{2}, 4.736 ; p, 0.030\right)$, while $i n h A-$ $15 \mathrm{~T}$ mutations did not differ significantly between these two major drug susceptibility groups (20.8\%.vs.21.8\%; $\chi^{2}$, $0.020 ; p, 0.887)$.

The resistance to RIF was due to mutations in the $r p o B$ gene in 49 of 53 MDR-TB isolates. A singlenucleotide substitution in position 516,526 and 531 accounted for $7.5 \%, 30.2 \%$ and $58.5 \%$ respectively of MDR-TB. The mutations in rpoB gene included 516 Tyr (3/53), 516Val (2/53), 526Arg (4/53), 526Tyr (11/53) and 531Leu (31/53). Double-spot mutations were presented in two MDR isolates, one with $516 \mathrm{Tyr} / 531 \mathrm{Leu}$ mutations and the other with $516 \mathrm{Val} / 531 \mathrm{Leu}$ mutations.

Table 2 Genetic mutations and their frequencies related to INH resistance in MTB isolates from the study sites

\begin{tabular}{|c|c|c|c|c|c|c|c|}
\hline \multirow{2}{*}{$\begin{array}{l}\text { Drug resistance } \\
\text { Profile }\end{array}$} & \multirow[t]{2}{*}{ Total } & \multicolumn{3}{|c|}{ katG315Ser $\rightarrow^{*}$} & \multirow{2}{*}{$\frac{\operatorname{inh} A-15 C \rightarrow^{*}}{\mathrm{~T}}$} & \multirow{2}{*}{$\begin{array}{c}\text { katG + } \\
\operatorname{inh}^{\S} A^{\S}\end{array}$} & \multirow[t]{2}{*}{ wt } \\
\hline & & Arg & Asn & Thr & & & \\
\hline$H$ & 55 & 2 & 1 & 28 & 12 & 0 & 12 \\
\hline \multicolumn{8}{|l|}{ MDR-TB } \\
\hline$H R$ & 32 & 0 & 1 & 19 & 6 & 1 & 5 \\
\hline HRS & 12 & 0 & 0 & 9 & 2 & 1 & 0 \\
\hline HRE & 5 & 0 & 0 & 5 & 0 & 0 & 0 \\
\hline HRSE & 4 & 0 & 1 & 2 & 1 & 0 & 0 \\
\hline \multicolumn{8}{|l|}{ PDR-TB } \\
\hline HS & 13 & 0 & 0 & 5 & 3 & 0 & 5 \\
\hline $\mathrm{HE}$ & 2 & 0 & 0 & 0 & 1 & 0 & 1 \\
\hline HSE & 8 & 0 & 1 & 5 & 1 & 0 & 1 \\
\hline
\end{tabular}

NOTE: $H$, isoniazid; $R$, rifampicin; $S$, streptomycin; $E$, ethambutol. MDR-TB, multidrug-resistant tuberculosis; PDR-TB, polydrug-resistant tuberculosis.

*: No. of isolates with mutations in single gene.

$\S$ : No. of isolates with mutations in multiple genes. Two INH resistant isolates shared the mutation in katG and inhA gene simultaneously: one with katG315Thr and inhA-15T mutations and the other with katG315Arg and inhA$15 \mathrm{~T}$ mutation. 
No $r p o B$ mutations were observed in isolates susceptible to RIF.

MTB clades designations were available for 122 of 131 isolates, with the following distribution of the genotypic lineages (Table 3): Beijing family (105/131 or $80.1 \%)$, Family 33 (8/131 or $6.1 \%)$, T lineage (7/131 or $5.3 \%)$, Haarlem (1/131 or $0.8 \%)$ and LAM (1/131 or $0.8 \%)$. Of the remaining 9 isolates, 6 had the spoligotyping pattern similar to $\mathrm{T}$ lineage, 1 similar to Family 33, 1 similar to Haarlem and 1 similar to LAM.

In the clustering analysis of INH resistant isolates specific to counties (Figure 1), IS6110 RFLP identified all together 94 IS6110 RFLP patterns, including 11 cluster patterns (27 isolates), 28 unique patterns/isolates in Deqing, 15 cluster patterns (36 isolates) and 40 unique patterns/isolates in Guanyun. The genotype and phenotype patterns of drug resistant TB were further investigated among the clustered isolates. Ten clusters (25 isolates) from Deqing and 12 clusters (32 isolates) from Guanyun contained isolates with different phenotype and/or genotype of drug resistance. In these clusters, INH resistant isolates with the katG315Thr mutation had the highest occurrence (51/ 57 or $89.5 \%)$. Additionally, 31 of 53 MDR-TB isolates $(58.5 \%)$ were observed in 19 clusters, with all carrying

Table 3 Spoligotyping pattern and frequency of the MTB isolates included

\begin{tabular}{|c|c|c|c|c|}
\hline Octal designation & Clade* $^{*}$ & Probability $^{\S}$ & SIT & No. of isolates \\
\hline 000000000003771 & Beijing & 0.99 & 1 & 105 \\
\hline 577777777760771 & T1 & 0.99 & 334 & 2 \\
\hline 777777777760031 & $\mathrm{~T} 1$ & 0.99 & 239 & 1 \\
\hline 777777777760771 & $\mathrm{~T} 1$ & 0.99 & 53 & 4 \\
\hline 777777367730771 & $\mathrm{~T} 1$ & 0.79 & new & 1 \\
\hline 777727377730771 & $\mathrm{~T} 1$ & 0.79 & new & 1 \\
\hline 777777777730771 & $\mathrm{~T} 1$ & 0.79 & 2597 & 1 \\
\hline 757737377730771 & $\mathrm{~T} 1$ & 0.79 & new & 1 \\
\hline 773637377730771 & $\mathrm{~T} 1$ & 0.79 & new & 1 \\
\hline 773677777730771 & $\mathrm{~T} 1$ & 0.79 & new & 1 \\
\hline 777777777146741 & Family33 & 0.99 & new & 1 \\
\hline 577777633566731 & Family33 & 0.99 & new & 1 \\
\hline 776767671146771 & family33 & 0.99 & new & 1 \\
\hline 777773777731771 & family33 & 0.99 & new & 2 \\
\hline 777777771146771 & family33 & 0.99 & new & 1 \\
\hline 777737177733571 & Family33 & 0.99 & new & 1 \\
\hline 777737717731761 & Family33 & 0.99 & new & 1 \\
\hline 777777777630771 & Family33 & 0.70 & new & 1 \\
\hline 777777776000371 & Haarlem 1 & 0.98 & 1498 & 1 \\
\hline 577777777700771 & Haarlem3 & 0.77 & new & 1 \\
\hline 777777603560731 & LAM9 & 0.99 & new & 1 \\
\hline 677777607560771 & LAM1 & 0.51 & 1755 & 1 \\
\hline
\end{tabular}

NOTE: SIT, spoligotype international type.

*: Spotclust program-assigned clade.

$\S$ : Probability that the spoligotyping pattern belongs to the clade. the katG315Thr mutation as well as the mutations either in position 516,526 or 531 of the $r p o B$ gene. In these clusters containing MDR-TB isolates, 18 clusters had INH-mono resistant and/or polydrug-resistant TB isolates simultaneously.

Binary logistic regression was applied to analyze the "clustering" of INH resistant isolates in association with patients' demographics, clinic profiles and the bacteriologic features (Table 4). Smear-positive TB patients had a significantly higher proportion of clustered INH resistant MTB strain than smear-negative patients $(54.9 \%$. vs.36.7\%; adjusted OR, 2.286; $p, 0.032$; 95\%CI,1.075$4.863)$, as well as the previously diagnosed patients when compared to newly diagnosed patients $(60.8 \%$. vs.40.0\%; adjusted OR, 2.159; $p, 0.040$; 95\%CI,1.0374.495). A significant higher proportion of MDR-TB isolates was clustered compared to INH mono resistant isolates (58.5\%.vs.38.2\%; adjusted OR, 2.384; $p, 0.030$; 95\%CI, 1.089-5.220). Compared with the wild type (wt) isolates, the isolates with the katG315Thr mutation were more likely to be clustered (73.0\%.vs.18.0\%; adjusted OR, $12.70 ; p, 0.001 ; 95 \% \mathrm{CI}, 6.357-14.80)$. The Beijing family isolates was mainly observed in clusters (56.2\%.vs.15.4\%; adjusted OR, 5.289; $p, 0.001 ; 95 \% \mathrm{CI}, 1.832-15.26)$. The INH resistant inhA-15T mutant isolates were more likely to be "unique" compared to those inhA-15 wild type isolates (14.3\%.vs.57.3\%; adjusted OR, 0.120; p, 0.001; 95CI\%, 0.038-0.375).

To further investigate the characteristics of katG315Thr mutant, the host and bacteriological variable were compared between the INH resistant isolates with the katG315Thr mutation group and those with other mutation/wild type group (Table 5). The katG315Thr mutant isolates had a significantly higher proportion of MDR-TB (48.6\%.vs.29.8\%; $p, 0.03$ ), the relevant mutation in $r p o B$ gene (47.3\%.vs.24.6\%, $p, 0.008)$ compared to other mutation/wild type group. And meanwhile, this allele was more likely to be clustered (73.0\%.vs.15.8\%; $p, 0.001)$ and belonged to the Beijing family $(87.8 \% . v s .70 .2 \%$; $p, 0.012)$.

\section{Discussion}

This study attempted to gain further insight into the molecular basis of the INH resistant MTB circulating in the rural area of eastern China. Transmission of INH resistant $\mathrm{MTB}$ and its possible contribution to the epidemic of MDR-TB were also a concern. The majority of INH strains from TB dispensaries in the rural Chinese area, exhibited INH mono-resistance and the double-drug-resistance profile especially including extra resistance to $\operatorname{RIF}(53 / 131)$ and STR (37/131). These resistance profiles corroborated the pattern of acquisition of resistance to extra drug resistance especially for RIF and STR in INH resistant isolates [1]. 


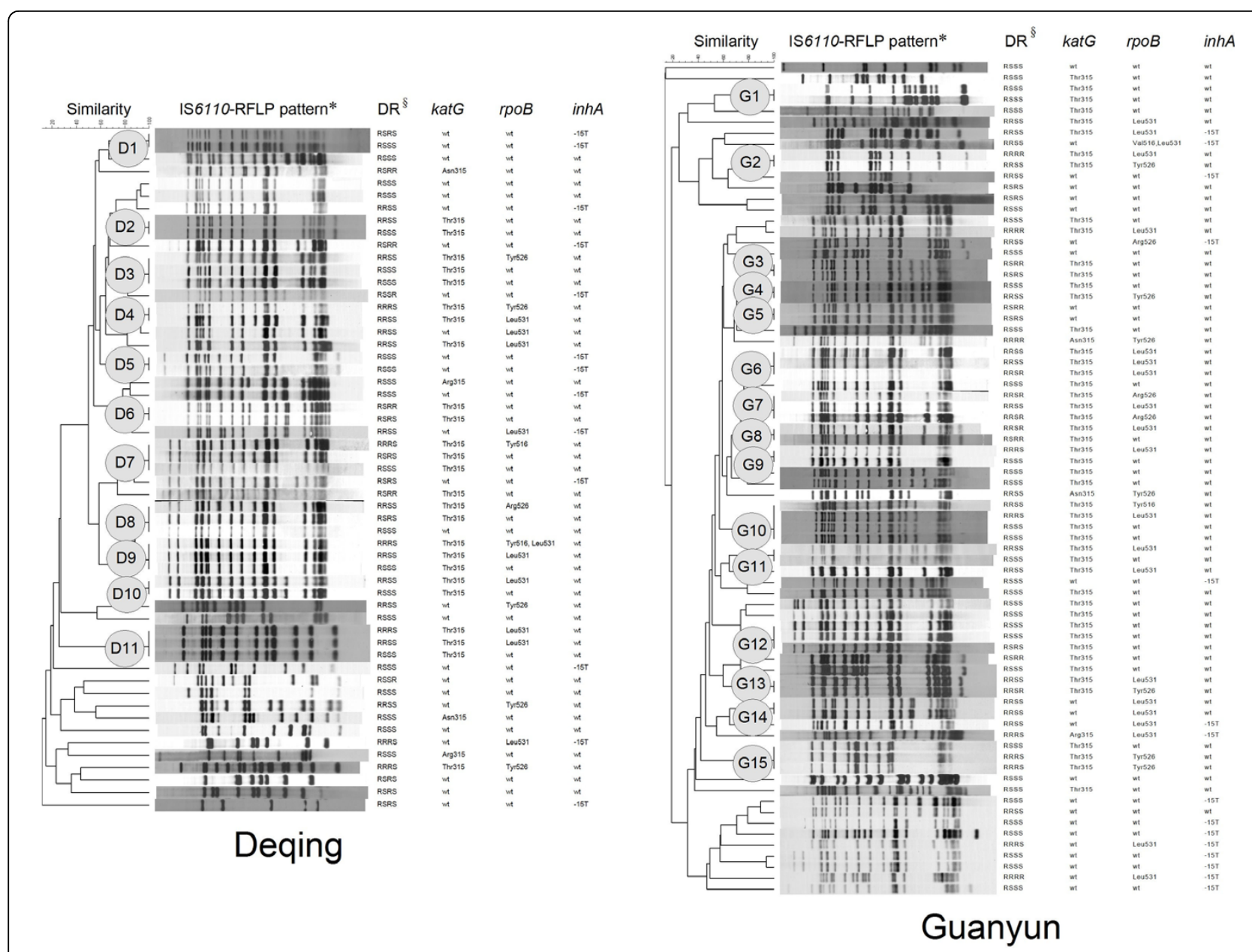

Figure 1 The phenotypes and genotypes of INH resistant MTB isolates with cluster and unique pattern respectively in Deqing and Guanyun. NOTE. DR, $1^{\text {st }}$ line Drug resistance profile. *: The circle contained the code for the cluster. §: Sequence of drugs was isoniazid, rifampicin, streptomycin, ethambutol; $R$, resistant; S, susceptible.

The prevalence of the katG315Thr mutation in MTB strains around the world varies, especially with regard to the prevalence of TB. High TB burden regions [15-17] always observe a higher prevalence of the katG315Thr mutation in INH resistant strain compared to low TB burden areas [18-20]. The present study also demonstrated a high prevalence of the katG $315 \mathrm{Thr}$ mutant allele in eastern rural China in around $50 \%$ of INH resistant isolates, which is consistent with the other report from China [21]. Furthermore in the current study, a significant correlation was confirmed between the common katG315Thr mutation in INH resistant strains and the presence of resistance to other drugs, especially for RIF. These observations might suggest that the INH resistant strain with the katG315Thr mutation may be more likely to develop the resistance to other $1^{\text {st }}$ line drugs especially for RIF [22-24] than the strains with other forms of INHresistance. Meanwhile, the high proportion of INH resistant katG 315Thr mutant allele bore the mutation in
rрoB gene, which was related to RIF resistance reported in China [25] and could be the reasons for the high risk of developing MDR in this allele.

The extensive transmission of the INH resistant MTB strain was observed in rural areas of eastern China, with $48.1 \%$ of the INH resistant MTB isolates in clusters. Most of INH resistant isolates with katG mutations were in clusters compared to wild type INH resistant isolates, suggesting its strong transmissibility, full virulence and survival advantage under the drug pressure in the host. This could be explained by the maintenance of the 30\% 40\% catalase-peroxidase activity in the INH resistant strain with the katG $315 \mathrm{Thr}$ mutation $[5,6]$ compared to other mutations related to drug resistance. Consequently, this allele was suspected as the cause of some earlier reported outbreaks [26-28] of INH resistant $\mathrm{TB}$ in specific areas and populations. Furthermore, the current study observed that in a cluster containing strains with different drug resistance profile and 
Table 4 Binary logistic regression analysis on the associations between demographical, clinical and bacteriological characteristics and clustering of INH resistant MTB isolates in the present study

\begin{tabular}{|c|c|c|c|c|c|c|c|}
\hline \multicolumn{2}{|c|}{ Variables } & \multicolumn{2}{|c|}{ INH resistant isolates } & \multirow{2}{*}{$\begin{array}{c}\text { unadjusted } \\
\text { OR }\end{array}$} & \multirow{2}{*}{$\begin{array}{l}\text { adjusted } \\
\mathrm{OR}^{*}\end{array}$} & \multirow[t]{2}{*}{$p$} & \multirow[t]{2}{*}{$95 \% \mathrm{Cl}$} \\
\hline & & No. patients & $n$ (\%) clustered & & & & \\
\hline \multicolumn{8}{|c|}{ Social-demographic and clinical characteristics: } \\
\hline \multirow[t]{3}{*}{ Age (year) } & $18 \sim$ & 34 & 12(35.3) & 1 & & & \\
\hline & $30 \sim$ & 65 & $34(52.3)$ & 2.011 & & & \\
\hline & $60 \sim$ & 32 & $17(53.1)$ & 2.078 & & & \\
\hline \multirow[t]{2}{*}{ Sex } & Female & 46 & $22(47.8)$ & 1 & & & \\
\hline & Male & 85 & $41(48.2)$ & 1.163 & & & \\
\hline \multirow[t]{2}{*}{ County } & Deqing & 55 & $27(49.1)$ & 1 & & & \\
\hline & Guanyun & 76 & $36(47.4)$ & 0.933 & & & \\
\hline BMI & $\geq 18.5$ & 94 & $41(43.6)$ & 1 & 1 & & \\
\hline Index & $<18.5$ & 37 & $22(59.5)$ & 1.896 & 1.833 & 0.13 & $0.836-4.019$ \\
\hline Treatment & New & 80 & $32(40.0)$ & 1 & 1 & & \\
\hline history & Previously & 51 & $31(60.8)$ & 2.325 & 2.159 & $0.040^{\S}$ & $1.037-4.495$ \\
\hline Sputum & negative & 49 & $18(36.7)$ & 1 & 1 & & \\
\hline smear & positive & 82 & $45(54.9)$ & 2.095 & 2.286 & $0.032^{\S}$ & $1.075-4.863$ \\
\hline \multirow[t]{2}{*}{ Cavity } & No & 111 & $50(45.0)$ & 1 & 1 & & \\
\hline & Yes & 20 & 13(65.0) & 2.266 & 2.423 & 0.091 & $0.868-6.761$ \\
\hline \multicolumn{8}{|c|}{ Bacteriological features: } \\
\hline Drug & INH mono & 55 & $21(38.2)$ & 1 & 1 & & \\
\hline \multirow[t]{2}{*}{ resistance } & MDR-TB & 53 & $31(58.5)$ & 2.281 & 2.384 & $0.030^{\S}$ & $1.089-5.220$ \\
\hline & $P D R-T B$ & 23 & $11(47.8)$ & 1.484 & 1.627 & 0.348 & $0.588-4.496$ \\
\hline \multirow[t]{3}{*}{ katG } & wt & 50 & $9(18.0)$ & 1 & 1 & & \\
\hline & $315 T h r$ & 74 & $54(73.0)$ & 12.3 & 12.7 & $0.001^{\S}$ & $6.357-14.80$ \\
\hline & others & 7 & $0(0)$ & - & - & & \\
\hline \multirow[t]{2}{*}{$\operatorname{inh} A$} & $w t$ & 103 & $59(57.3)$ & 1 & 1 & & \\
\hline & $-15 T$ & 28 & $4(14.3)$ & 0.124 & 0.12 & $0.001^{\S}$ & $0.038-0.375$ \\
\hline Beijing & No & 26 & $4(15.4)$ & 1 & 1 & & \\
\hline genotype & Yes & 105 & $59(56.2)$ & 5.183 & 5.289 & $0.001^{\S}$ & $1.832-15.26$ \\
\hline
\end{tabular}

NOTE. OR, odds ratio; 95\%Cl, confidence interval; wt, wild type; MDR-TB, multidrug-resistant tuberculosis; PDR-TB, polydrug-resistant tuberculosis.

*: OR and $95 \% \mathrm{Cl}$ were adjusted by age, sex and county of subjects in binary logistic regression model.

§: $p<0.05$.

resistance conferring mutations, the INH resistance was caused by this mutation. This increased the possibility that the INH resistant MTB strain with the katG 315 mutation in the index person was more likely to experience a series of subsequent mutations, leading to the accumulation of extra drug resistance and the development of MDR- and/or extreme drug resistant(XDR)-TB [29]. The accumulation of drug resistance could be accredited by less efficiencies of standard chemotherapy, the subsequent mutation of drug resistance-related gene and selective survival of drug resistant strain during the recent transmission.

Apart from the role of the katG315Thr mutation, the transmissibility of INH resistant strain might be related to endemic characteristics of the Beijing family in rural China. The Beijing genotype is apparently the most prevalent genotype in China, representing of $80.2 \% \mathrm{INH}$ resistant and $83 \%$ MDR-TB isolates in the current study. Our study also found that the MTB strain from the Beijing family was also the main source of recent transmission causing INH resistant TB. The Beijing genotype has been reported to be associated with treatment failure and relapse. Although the reasons for this are not known, it appears that the infections with the Beijing genotype strain may be more persistent, probably leading to longer duration of infectiousness and creating a better opportunity for transmission under the anti-TB drug pressure [30].

Additionally in the current study, INH resistant MTB was more likely to be clustered in smear-positive and previously diagnosed patients. The smear-positive status might extend the transmission period of the pathogen between hosts and allow MTB to attack more people in the surroundings of the index case. Although the previous TB could be cured by direct observed chemotherapy, those formerly treated cases could still be at risk 
Table 5 Comparison of host and bacteriological characteristics between isoniazid resistant isolates with katG315Thr mutations and other mutation/wild type

\begin{tabular}{|c|c|c|c|c|c|}
\hline & & \multicolumn{2}{|c|}{ No. of isolates with: } & \multirow[b]{2}{*}{$\chi^{2}$} & \multirow[b]{2}{*}{$p$} \\
\hline \multicolumn{2}{|c|}{ Variables } & katG315Thr $n=74$ & Others $n=57$ & & \\
\hline \multirow[t]{2}{*}{ Sputum smear } & Negative & $23(31.1)$ & $26(45.6)$ & 2.905 & 0.088 \\
\hline & Positive & $51(68.9)$ & $31(54.4)$ & & \\
\hline \multirow[t]{2}{*}{ Treatment history } & New & $41(55.4)$ & $39(68.4)$ & 2.294 & 0.13 \\
\hline & Previously & $33(44.6)$ & 18(31.6) & & \\
\hline \multirow[t]{2}{*}{ Cavity } & No & 59(79.7) & $52(91.2)$ & 3.291 & 0.07 \\
\hline & Yes & 15(20.3) & $5(8.8)$ & & \\
\hline \multirow[t]{2}{*}{ Drug resistance profile } & Others & $38(51.4)$ & $40(70.2)$ & 4.736 & $0.030^{*}$ \\
\hline & $M D R-T B$ & $36(48.6)$ & 17(29.8) & & \\
\hline \multirow[t]{2}{*}{ rpoB mutation } & No & $39(52.7)$ & $43(75.4)$ & 7.11 & $0.008^{*}$ \\
\hline & Yes & $35(47.3)$ & $14(24.6)$ & & \\
\hline \multirow[t]{2}{*}{ Clustered } & No & $20(27.0)$ & 48(84.2) & 42.18 & $0.001^{*}$ \\
\hline & Yes & $54(73.0)$ & $9(15.8)$ & & \\
\hline \multirow[t]{2}{*}{ Beijing family } & No & $9(12.2)$ & 17(29.8) & 6.314 & $0.012^{*}$ \\
\hline & Yes & 65(87.8) & $40(70.2)$ & & \\
\hline
\end{tabular}

$*: p<0.05$

for the TB re-infection from recent transmissions since the risk factors for TB (low socio-economic status, exposure to MTB etc.) are still present. In combination with these conditions, the risk of INH resistant MTB transmission between hosts increases.

The epidemic of MDR-TB can occur as a result of the spontaneous mutations in MTB and selection under suboptimal drug therapy or extensive transmission of the drug resistant $\mathrm{MTB}$, or both combined. In the current study, a significant high proportion of MDR-TB isolates were observed in clusters compared to the other form of INH mono-resistant isolates. Most clusters with MDR-TB isolates also contained isolates with other drug resistance profiles including INH mono-resistance and poly drug-resistant tuberculosis simultaneously. Based on these observations, the epidemic of MDR-TB in rural China could be explained by two possibilities: 1) the epidemic of MDR-TB might result from the recent transmission of MDR-TB strains in an area ridden by INH resistant MTB. 2) Recent transmission of the drug resistant MTB and the selection of MTB under drug pressure might exert the symbiotic interaction in an epidemic of MDR-TB. The combination of INH resistance and maintained virulence might make it possible for some INH resistant strains, especially those with the katG315Thr mutation, to acquire extra drug resistance and become MDR-TB. It deserves further investigation to determine which mechanism may play the critical role in the epidemic of MDR-TB, since the implication behind it could be meaningful to evaluate the performance of local TB control as well as to determine the MDR-TB control strategies suitable for rural areas of China and as well as other similar high burden settings.

\section{Conclusions}

INH resistant MTB was transmitted widely in eastern rural areas of China. Also the correlation of prevalence and transmission between INH resistant isolates especially with the katG315Thr mutation and MDR-TB was confirmed. Therefore, it is important to recognize the katG315Thr mutants among INH-resistant strains, which could be seen as a risk factor for subsequent development of MDR-TB. Early detection of the patients with INH resistant strains would facilitate the modification of treatment regimens and appropriate infection control measures can be taken in time to reduce the risk of further development and transmission of MDR-TB.

List of Abbreviations

MTB: Mycobacterium tuberculosis; MDR: multidrug-resistant; INH: isoniazid; RIF: rifampicin; STR: streptomycin; EMB: ethambutol; RFLP: restriction fragment length polymorphism; SIT: spoligotype international type.

\section{Acknowledgements}

We wish to thank health authorities and investigators in Deqing county and Guanyun county for their cooperation. This study was supported by grants (PI, Biao Xu; No. 30771843) from National Natural Science Foundation of China, the National Key Project for Infectious Disease (No.2008ZX10003-015) and Shanghai Leading Academic Discipline Project (No. B118).

\section{Author details}

'Department of Epidemiology, School of Public Health, Fudan University, Shanghai, China. ${ }^{2}$ Department of Bacteriology, Swedish Institute for Infectious Disease Control, Stockholm, Sweden.

\section{Authors' contributions}

$\mathrm{YH}$ carried out the data collection and molecular genotyping studies, participated in the PCR and sequence alignment and drafted the manuscript. SH has revised it critically for important intellectual content. WJ was involved in all the microbiological research. WW participated in the conception and design, acquisition of data, its analysis and interpretation. BX conceived the study, developed the design, coordinated the 
implementation, and helped to revise the manuscript. All authors read and approved the final manuscript.

\section{Competing interests}

The authors declare that they have no competing interests.

Received: 9 November 2009 Accepted: 28 February 2010 Published: 28 February 2010

\section{References}

1. Espinal MA, Laszlo A, Simonsen L, Boulahbal F, Kim SJ, Reniero A, Hoffner S, Rieder $\mathrm{HL}$, Binkin N, Dye C: Global trends in resistance to antituberculosis drugs. World Health Organization-International Union against Tuberculosis and Lung Disease Working Group on Anti-Tuberculosis Drug Resistance Surveillance. N Engl J Med 2001, 344:1294-1303.

2. Aziz MA, Wright A, Laszlo A, De Muynck A, Portaels F, Van Deun A, Wells C, Nunn P, Blanc L, Raviglione M: Epidemiology of antituberculosis drug resistance (the Global Project on Anti-tuberculosis Drug Resistance Surveillance): an updated analysis. Lancet 2006, 368:2142-2154.

3. WHO: The WHO/IUATLD Global project on anti-tuberculosis drug resistance surveilance. Anti-tuberculosis drug resistance in the world. Report No.4 WHO/HTM/TB/2008394. Geneva, Switzerland.

4. Dye C, Espinal MA: Will tuberculosis become resistant to all antibiotics? Proc Biol Sci 2001, 268:45-52.

5. Zhang Y, Heym B, Allen B, Young D, Cole S: The catalase-peroxidase gene and isoniazid resistance of Mycobacterium tuberculosis. Nature 1992, 358:591-593.

6. Banerjee A, Dubnau E, Quemard A, Balasubramanian V, Um KS, Wilson T, Collins D, de Lisle G, Jacobs WR Jr: inhA, a gene encoding a target for isoniazid and ethionamide in Mycobacterium tuberculosis. Science 1994, 263:227-230

7. Heym B, Alzari PM, Honore N, Cole ST: Missense mutations in the catalase-peroxidase gene, katG, are associated with isoniazid resistance in Mycobacterium tuberculosis. Mol Microbiol 1995, 15:235-245.

8. Musser JM, Kapur V, Williams DL, Kreiswirth BN, van Soolingen D, van Embden JD: Characterization of the catalase-peroxidase gene (katG) and inhA locus in isoniazid-resistant and -susceptible strains of Mycobacterium tuberculosis by automated DNA sequencing: restricted array of mutations associated with drug resistance. J Infect Dis 1996, 173:196-202

9. Zhang $M$, Yue J, Yang YP, Zhang HM, Lei JQ, Jin RL, Zhang $X L$, Wang $H H$ : Detection of mutations associated with isoniazid resistance in Mycobacterium tuberculosis isolates from China. J Clin Microbiol 2005, 43:5477-5482

10. van Soolingen D, Qian L, de Haas PE, Douglas JT, Traore H, Portaels F, Qing HZ, Enkhsaikan D, Nymadawa P, van Embden JD: Predominance of a single genotype of Mycobacterium tuberculosis in countries of east Asia. J Clin Microbiol 1995, 33:3234-3238.

11. Hu Y, Mathema B, Wang W, Hoffner S, Kreiswirth B, Xu B: Prevalence of multidrug-resistant pulmonary tuberculosis in counties with different duration of DOTS implementation in rural China. Microb Drug Resist 2008, 14:227-232.

12. Canetti G, Froman S, Grosset J, Hauduroy P, Langerova M, Mahler HT, Meissner G, Mitchison DA, Sula L: Mycobacteria: Laboratory Methods for Testing Drug Sensitivity and Resistance. Bull World Health Organ 1963, 29:565-578.

13. van Embden JD, Cave MD, Crawford JT, Dale JW, Eisenach KD, Gicquel B, Hermans P, Martin C, McAdam R, Shinnick TM: Strain identification of Mycobacterium tuberculosis by DNA fingerprinting: recommendations for a standardized methodology. J Clin Microbiol 1993, 31(2):406-409.

14. Vitol I, Driscoll J, Kreiswirth B, Kurepina N, Bennett KP: Identifying Mycobacterium tuberculosis complex strain families using spoligotypes. Infect Genet Evol 2006, 6(6):491-504.

15. Dobner P, Rusch-Gerdes S, Bretzel G, Feldmann K, Rifai M, Loscher T, Rinder $\mathrm{H}$ : Usefulness of Mycobacterium tuberculosis genomic mutations in the genes katG and inhA for the prediction of isoniazid resistance. Int J Tuberc Lung Dis 1997, 1:365-369.

16. Escalante P, Ramaswamy S, Sanabria H, Soini H, Pan X, Valiente-Castillo O, Musser JM: Genotypic characterization of drug-resistant Mycobacterium tuberculosis isolates from Peru. Tuber Lung Dis 1998, 79:111-118.
17. Marttila HJ, Soini H, Eerola E, Vyshnevskaya E, Vyshnevskiy BI, Otten TF, Vasilyef AV, Viljanen MK: A Ser315Thr substitution in KatG is predominant in genetically heterogeneous multidrug-resistant Mycobacterium tuberculosis isolates originating from the St. Petersburg area in Russia. Antimicrob Agents Chemother 1998, 42:2443-2445.

18. Lee $A S$, Lim $I H$, Tang $L L$, Telenti A, Wong SY: Contribution of kasA analysis to detection of isoniazid-resistant Mycobacterium tuberculosis in Singapore. Antimicrob Agents Chemother 1999, 43:2087-2089.

19. Varela G, Gonzalez S, Gadea P, Coitinho C, Mota I, Gonzalez G, Goni F Rivas C, Schelotto F: Prevalence and dissemination of the Ser315Thr substitution within the KatG enzyme in isoniazid-resistant strains of Mycobacterium tuberculosis isolated in Uruguay. J Med Microbiol 2008, 57:1518-1522.

20. Fang Z, Doig C, Rayner A, Kenna DT, Watt B, Forbes KJ: Molecular evidence for heterogeneity of the multiple-drug-resistant Mycobacterium tuberculosis population in Scotland (1990 to 1997). J Clin Microbiol 1999, 37:998-1003.

21. Chen $X, M a ~ Y$, Jin $Q$, Jiang GL, Li CY, Wang Q: Characterization of the katG, inhA, ahpC, kasA, and oxyR gene mutations in isoniazid-resistant and susceptible strain of Mycobacterium tuberculosis by automated DNA sequencing. Zhonghua Jie He He Hu Xi Za Zhi 2005, 28:250-253.

22. Bakonyte D, Baranauskaite A, Cicenaite J, Sosnovskaja A, Stakenas P. Molecular characterization of isoniazid-resistant Mycobacterium tuberculosis clinical isolates in Lithuania. Antimicrob Agents Chemother 2003, 47:2009-2011.

23. Hillemann D, Kubica T, Agzamova R, Venera B, Rusch-Gerdes S, Niemann S: Rifampicin and isoniazid resistance mutations in Mycobacterium tuberculosis strains isolated from patients in Kazakhstan. Int J Tuberc Lung Dis 2005, 9:1161-1167.

24. Piatek AS, Telenti A, Murray MR, El-Hajj H, Jacobs WR Jr, Kramer FR, Alland D: Genotypic analysis of Mycobacterium tuberculosis in two distinct populations using molecular beacons: implications for rapid susceptibility testing. Antimicrob Agents Chemother 2000, 44:103-110.

25. Huang $H$, Jin $Q, M a Y, C$ hen $X$, Zhuang $Y$ : Characterization of rpoB mutations in rifampicin-resistant Mycobacterium tuberculosis isolated in China. Tuberculosis (Edinb) 2002, 82:79-83.

26. Ahmad S, Fares E: Genotypic diversity among isoniazid-resistant isolates of Mycobacterium tuberculosis from Rashid hospital in Dubai, United Arab Emirates. Med Princ Pract 2005, 14:16-21.

27. Caws M, Duy PM, Tho DQ, Lan NT, Hoa DV, Farrar J: Mutations prevalent among rifampin- and isoniazid-resistant Mycobacterium tuberculosis isolates from a hospital in Vietnam. J Clin Microbio/ 2006, 44:2333-2337.

28. Mokaddas E, Ahmad S, Abal AT: Molecular fingerprinting of isoniazidresistant Mycobacterium tuberculosis isolates from chest diseases hospital in Kuwait. Microbiol Immunol 2002, 46:767-771.

29. Ano H, Matsumoto $T$, Suetake $T$, Nagai T, Tamura $Y$, Takamatsu I, Iwasaki T, Matsuoka H, Sasada S, Tetsumoto S: Relationship between the isoniazidresistant mutation katGS315T and the prevalence of MDR-/XDR-TB in Osaka, Japan. Int J Tuberc Lung Dis 2008, 12:1300-1305.

30. Kruuner A, Hoffner SE, Sillastu H, Danilovits M, Levina K, Svenson SB, Ghebremichael S, Koivula T, Kallenius G: Spread of drug-resistant pulmonary tuberculosis in Estonia. J Clin Microbiol 2001, 39:3339-3345.

\section{Pre-publication history}

The pre-publication history for this paper can be accessed here: [http://www.biomedcentral.com/1471-2334/10/43/prepub]

doi:10.1186/1471-2334-10-43

Cite this article as: Hu et al:: Extensive transmission of isoniazid resistant $M$. tuberculosis and its association with increased multidrugresistant TB in two rural counties of eastern China: A molecular epidemiological study. BMC Infectious Diseases 2010 10:43. 